\title{
Metodologias para avaliação do comportamento ingestivo de novilhas suplementadas a pasto ${ }^{1}$
}

\author{
Methodologies for evaluation of ingestive behavior of heifers \\ supplemented in grazing
}

\author{
Hermógenes Almeida de Santana Junior ${ }^{2 *}$; Robério Rodrigues Silva ${ }^{3}$; \\ Gleidson Giordano Pinto Carvalho ${ }^{4}$; Fabiano Ferreira Silva ${ }^{3}$; \\ Patrícia Barcelos Costa ${ }^{5}$; Fabricio Bacelar Lima Mendes ${ }^{6}$; \\ Alyson Andrade Pinheiro ${ }^{6}$; Elizangela Oliveira Cardoso Santana ${ }^{6}$; \\ George Abreu Filho ${ }^{6}$; Geraldo Trindade Júnior ${ }^{3}$
}

\section{Resumo}

O estudo foi realizado com o objetivo de comparar diferentes metodologias para avaliação do comportamento ingestivo de novilhas suplementadas a pasto. $\mathrm{O}$ experimento foi conduzido na fazenda Princesa do Mateiro, município de Ribeirão do Largo, Bahia. Foram utilizadas 30 novilhas com grau de sangue 5/8 Guzerá linhagem leiteira e 3/8 Holandesa, com média de 18 meses de idade e peso corporal médio de $187 \pm 13,1 \mathrm{~kg}$. Os tratamentos foram: Duração de avaliação (24, 12 e oito horas); Intervalos de observação (10, 20 e 30 minutos); Repetições por observação (três, duas e uma); Turnos de observação (dois e um). A avaliação do comportamento foi realizada no $25^{\circ}, 26^{\circ}, 27^{\circ}$ e $28^{\circ}$ dia de cada período, totalizando então quatro avaliações. Os percentuais da atividade de pastejo da duração de avaliação $8 \mathrm{~h}$ e $12 \mathrm{~h}$ diferiram da duração de avaliação padrão de $24 \mathrm{~h}(\mathrm{P}<0,05)$. A duração de avaliação em testes ( $8 \mathrm{~h}$ e $12 \mathrm{~h}$ ) apresentou diferenças da duração de avaliação de 24 horas para os percentuais de ruminação $(\mathrm{P}<0,05)$. Já para os percentuais de outras atividades, não foi verificado diferenças entre a duração de avaliação em testes e a padrão $(\mathrm{P}>0,05)$. Para o tempo de pastejo, ruminação, alimentação no cocho, outras atividades, alimentação total e mastigação total não foram verificados diferenças estatísticas entre os intervalos de observações 20 e 30 minutos quando comparados com o de 10 minutos $(\mathrm{P}>0,05)$. A duração de avaliação de 24 horas é a recomendada para avaliação do comportamento ingestivo em termos de parâmetros nutricionais e metabólicos, pois as demais promovem distorção dos dados em função da variação da intensidade das atividades ao longo do dia.

Palavras-chave: Bocado, bovino, consumo, métodos, pastejo

\footnotetext{
${ }^{1}$ Projeto financiado pelo Banco do Nordeste do Brasil, BNB, Fortaleza, CE.

${ }^{2}$ Prof. Adjunto da Universidade Estadual do Piauí, UESPI, Campus Jesualdo Cavalcanti, Corrente, PI. E-mail: hsantanajunior@, hotmail.com

${ }^{3}$ Profs. Adjuntos da Universidade Estadual do Sudoeste da Bahia, UESB, Campus Juvino Oliveira. Itapetinga, BA. E-mail: rrsilva@uesb.com.br; ffsilva@cnpq.br; geraldouesb@hotmail.com

${ }^{4}$ Prof. Adjunto da Universidade Federal da Bahia, UFBA, Salvador, BA. E-mail: gleidsongiordano@yahoo.com.br

${ }^{5}$ Prof Adjunta da Universidade Estadual do Oeste do Paraná, UNIOESTE, Marechal Cândido Rondon, PR. E-mail: costa222@ hotmail.com

${ }^{6}$ Discentes do Curso de Mestrado e Doutorado em Produção de Ruminantes no Programa de Pós-Graduação em Zootecnia, UESB, Campus Juvino Oliveira. Itapetinga, BA. E-mail: fabricio.bacelar@hotmail.com; jagualyson@bol.com.br; eocardoso@hotmail. com; georgeabreu@hotmail.com

* Autor para correspondência
} 


\begin{abstract}
The study was conducted to compare different methodologies for evaluation of ingestive behavior of heifers supplemented in grazing. The experiment was conducted at Princesa do Mateiro farm, Ribeirao do Largo, Bahia. Were used 30 heifers with blood level 5/8 Guzera milk lineage and 3/8 Holstein, with an average of 18 months of age and body weight of $187 \pm 13.1 \mathrm{~kg}$. The treatments were: Duration of avaliation (24, 12 and eight hours) of observation intervals (10,20 and 30 minutes); repetitions for observation (three, two and one) shifts of observation (two and one). The behavior evaluation was performed on day $25^{\text {th }}, 26^{\text {th }}, 27^{\text {th }}$ and $28^{\text {th }}$ of each period, then a total of four ratings. The percentage of grazing activity on the duration of $8 \mathrm{~h}$ and $12 \mathrm{~h}$ evaluation differed from the standard evaluation period of 24 hours $(\mathrm{P}<0.05)$. The duration of evaluation tests $(8 \mathrm{~h}$ and $12 \mathrm{~h})$ showed differences in duration of 24 hours to evaluate the percentages of rumination $(\mathrm{P}<0.05)$. As for the percentage of other activities, no difference was observed between duration evaluation and standard tests $(\mathrm{P}>0.05)$. For grazing time, rumination, feeding at the trough, other activities, total feeding and total chewing have not been verified statistical differences between the ranges of observations 20 and 30 minutes compared with 10 minutes $(\mathrm{P}<0.05)$. The duration of evaluation of 24 hours is recommended for evaluation of ingestive behavior in terms of nutritional and metabolic parameters, as other promote distortion of the data as a function of the intensity of activities throughout the day.
\end{abstract}

Key words: Bit, cattle, grazing, intake, methods

\section{Introdução}

$\mathrm{Na}$ última década, vários pesquisadores têm utilizado o estudo do comportamento ingestivo para nortear pesquisas com suplementação de bovinos a pasto, e através deste, quantificar os efeitos de estratégias de suplementação, níveis de suplementação e teores dos nutrientes no concentrado. Porém, no que tange as metodologias utilizadas para esses estudos, ocorre uma grande discrepância entre a duração de avaliação, intervalo de observações e número de turnos e repetições adequadas para obter dados com alta acurácia.

Sabe-se que a única duração de avaliação que expressa realmente o comportamento do animal, em termos de aspectos metabólicos, ao longo do dia é o de 24 horas, e que já foi utilizado por alguns autores (BREMM et al., 2008; FREITAS et al., 2010), entretanto existem outros autores que utilizaram o tempo de avaliação de 20 horas (OLIVO et al., 2006), 12 horas (ÍTAVO et al., 2008; SILVA et al., 2010), 11 horas (MACARI et al., 2007), 10 horas (PEREIRA et al., 2005; SOUZA et al., 2007) e 9 horas e 20 minutos (PARDO et al., 2003).

O mesmo ocorre com o intervalo de observação, pois desde a década de 70 , observou-se que os resultados do intervalo de cinco minutos eram semelhantes aos da avaliação contínua (GARY; SHERRITT; HALE, 1970), no entanto existem pesquisadores utilizando cinco minutos (ÍTAVO et al., 2008; FREITAS et al., 2010) e outros utilizando 10 minutos (BREMM et al., 2008; SILVA et al., 2008; BAGGIO et al., 2009). Verifica-se que o maior intervalo utilizado é o de 10 minutos, mas será que é possível a utilização de intervalos superiores. Percebe-se que na maioria dos trabalhos de observação do comportamento ingestivo de ruminantes, a escolha da escala do intervalo de tempo a ser utilizada tem sido feita de forma aleatória, o que pode comprometer os resultados e a precisão da pesquisa.

Alguns estudos mostram algumas tendências, como a possibilidade de observar animais pelo modelo Scan Sampling a até trinta minutos de intervalos, conforme proposto por Silva et al. (2005), que trabalharam com novilhas holandesas confinadas. Esses autores, entretanto, ressaltam que não há viabilidade desses tipos de estudos para discretização de séries temporais.

Objetivou-se avaliar as metodologias da duração, intervalo e repetições necessários para o 
estudo de comportamento ingestivo de novilhas a pasto.

\section{Material e Métodos}

$\mathrm{O}$ experimento foi conduzido na fazenda Princesa do Mateiro, município de Ribeirão do Largo, localizado na região sudoeste do estado da Bahia. Foram utilizadas 30 novilhas mestiças com grau de sangue 5/8 Guzerá linhagem leiteira e 3/8 Holandesa, com média de 18 meses de idade e peso corporal médio inicial de $187 \pm 13,1 \mathrm{~kg}$, distribuídas em 10 animais por tratamento. O experimento teve duração de 224 dias, tendo seu início em 25 de maio de 2008 e finalizando no dia 04 de janeiro de 2009, sendo composto por quatro períodos de 56 dias.

Todos os animais foram vermifugados e avaliados quanto ao seu estado sanitário no momento da seleção para inclusão no experimento. Os animais criados em pastejo com lotação rotacionada de Brachiaria brizantha cv. Marandú em área de 10 ha, divididos em quatro piquetes de áreas equivalentes, sendo que os mesmos foram vedados 30 dias antes do início do experimento.

Os animais foram suplementados com ração concentrada (Tabela 1), sendo que a mesma foi fornecida diariamente e sempre no mesmo horário, às $10 \mathrm{~h}$, em cochos plásticos, coletivo com duplo acesso, sem cobertura, com dimensionamento linear de $80 \mathrm{~cm} /$ animal.

Tabela 1. Proporções de ingredientes do concentrado por período, na base da matéria natural.

\begin{tabular}{lcccc}
\hline \multirow{2}{*}{ Ingrediente } & \multicolumn{3}{c}{ Período } \\
\cline { 2 - 5 } & $1^{\mathrm{o}}$ & $2^{\mathrm{o}}$ & $3^{\mathrm{o}}$ & $4^{\mathrm{o}}$ \\
\hline Milho grão moído & 28,20 & 66,47 & 70,52 & 72,05 \\
Farelo de soja & 34,08 & 26,75 & 25,92 & 25,21 \\
Farelo de trigo & 35,20 & 5,28 & 0,08 & --- \\
Calcário calcítico & 1,32 & --- & 0,67 & 0,84 \\
Fosfato bicálcico & --- & --- & 0,51 & --- \\
Ureia $_{\text {Sal mineral }^{1}}^{---}$ & --- & --- & 0,36 \\
\hline
\end{tabular}

${ }^{1}$ Composição: Cálcio $140 \mathrm{~g}$; fósforo $65 \mathrm{~g}$; sódio $148 \mathrm{~g}$; magnésio 5 g; enxofre 12 g; cobalto 107 mg; cobre 1550 mg; iodo 150 mg; manganês 1400 mg; níquel 30 mg; selênio 18 mg; zinco 4500 mg; 1120 mg; flúor (máximo) 650 mg.

Fonte: Elaboração dos autores.

Os animais foram identificados por meio de suas características morfológicas e brincos plásticos numerados.

Os tratamentos foram compostos de 10 animais cada, sendo um estudo de metodologia por dia de avaliação em cada período experimental. Os mesmos foram:

- Duração de avaliação (24, 12 e oito horas);

- Intervalos de observação (10, 20 e 30 minutos);

- Repetições por observação (três, duas e uma);
- Turnos de observação (dois e um).

Em cada período, a coleta de dados da duração de avaliação foi realizada no $25^{\circ}$ dia, os intervalos de observação foram realizados no $26^{\circ}$ dia, as repetições por observação foi realizada no $27^{\circ}$ dia, os turnos de observação foi realizada no $28^{\circ}$ dia, totalizando assim, quatro avaliações e 40 repetições por tratamento.

Os animais foram avaliados visualmente, por dois observadores treinados para cada grupo alimentar, sendo os mesmos, posicionados estrategicamente 
de forma a não incomodar os animais. Para saber o tempo gasto em cada atividade foram utilizados relógios digitais. Para o período noturno, não houve a necessidade de iluminação artificial, pois o período de coleta coincidiu com a fase de lua cheia em todas as avaliações (56 em 56 dias que se assemelha com 02 ciclos da lua).

As variáveis comportamentais estudadas foram: tempo de pastejo (PAS), tempo de ruminação (RUM), tempo de alimentação no cocho (COC) e tempo em outras atividades (OUT). As atividades comportamentais foram consideradas mutuamente excludentes, conforme definição de Pardo et al. (2003).

O tempo gasto pelos animais na seleção e apreensão da forragem, incluindo os curtos espaços de tempo utilizados no deslocamento para a seleção da forragem foi considerado tempo de pastejo (HANCOCK, 1953). O tempo de ruminação correspondeu aos processos de regurgitação, remastigação, reinsalivação e redeglutição. O tempo de alimentação no cocho foi o tempo despendido pelo animal no consumo de suplemento. Enquanto o tempo em outras atividades (descanso, consumo de água, interações, etc...), foram todas as atividades com exceção das citadas acima.

O tempo de alimentação total (TAT) e de mastigação total (TMT) foi determinado pelas equações abaixo:

$$
\mathrm{TAT}=\mathrm{PAS}+\mathrm{COC}
$$

em que: PAS (minutos) = tempo de pastejo; $\mathrm{COC}$ (minutos) $=$ tempo de alimentação no cocho.

$$
\mathrm{TMT}=\mathrm{PAS}+\mathrm{RUM}+\mathrm{COC}
$$

em que: PAS (minutos) = tempo de pastejo; RUM $($ minutos $)=$ tempo de ruminação; COC (minutos) $=$ tempo de alimentação no cocho.

A discretização das séries temporais foi realizada diretamente nas planilhas de coleta de dados, com a contagem dos períodos discretos de pastejo, ruminação, outras atividades e alimentação no cocho, conforme descrito por Silva et al. (2006). A duração média de cada um dos períodos discretos foi obtida pela divisão dos tempos diários de cada uma das atividades pelo número de períodos discretos da mesma atividade. Foram calculados as eficiências de alimentação da MS, FDN, NDT, CNF e PB e ruminação da MS e FDN.

Foram realizadas observações por dois períodos do dia (manhã e tarde), e com três repetições por período (BURGER et al., 2000), a fim de determinar o número de mastigações merícicas por bolo ruminado (MMB) e o tempo gasto para ruminação de cada bolo (TBo).

As variáveis, número de bolo ruminado por dia (BOL), velocidade de mastigação (VeM), tempo por mastigação merícica (TeM) e mastigações merícicas por dia (MMnd) foram calculadas pelas equações abaixo:

$$
\mathrm{BOL}=\mathrm{RUM} / \mathrm{TBo}
$$

em que: BOL em número por dia; RUM - tempo de ruminação em segundos por dia; TBo - tempo por bolo ruminado em segundos.

$$
\mathrm{VeM}=\mathrm{MMB} / \mathrm{TBo}
$$

em que: VeM em mastigações merícicas; MMB - número de mastigações merícicas por bolo; TBo tempo por bolo ruminado em segundos.

$$
\mathrm{TeM}=\mathrm{TBo} / \mathrm{MMB}
$$

em que: TeM em segundos; TBo - tempo por bolo ruminado em segundos; MMB - número de mastigações merícicas por bolo.

$$
\mathrm{MMnd}=\mathrm{BOL} * \mathrm{MMB}
$$

em que: MMnd em número por dia; BOL número de bolos ruminados por dia; $\mathrm{MMB}$ - número de mastigações merícicas por bolo.

Após obtenção dos dados, os mesmos foram divididos em três metodologias: Manhã e tarde (MANTAR, metodologia padrão) conforme SILVA et al. (2008) e outras duas metodologias em teste, sendo somente um turno de observação 
(pela manhã - MANHÃ ou pela tarde - TARDE), totalizando 60,30 e 30 observações por grupo de animais, respectivamente. Também foi avaliado a metodologia de repetição, avaliando com uma (1R, metodologia em teste), duas (2R, metodologia em teste) e três (3R, metodologia padrão segundo BURGER et al., 2000) repetições por turno, totalizando 20, 40 e 60 observações por grupo de animais, respectivamente. Após a obtenção dos resultados das variáveis testadas, todas foram comparadas com as médias das variáveis das metodologias validadas (padrão).

Durante os mesmos períodos de avaliação do comportamento animal, quando os animais estavam em atividade de pastejo a mais de 30 minutos, foi registrada a taxa de bocado $(\mathrm{TxB})$ dos animais de cada grupo alimentar, estimada por meio do tempo gasto pelo animal para realizar 20 bocados (HODGSON, 1982). Para o cálculo da massa de bocado (MaB), dividiu-se o consumo diário pelo total de bocados diários (JAMIESON; HODGSON, 1979).

Também foi coletado o número de bocados e o tempo desprendido por deglutição. Os resultados das observações de bocados e deglutição foram registrados em seis ocasiões durante o dia, conforme Baggio et al. (2009), sendo três avaliações durante a manhã e três à tarde, e usados também para determinar o número de bocados por dia (NBD), que é o produto entre taxa de bocado e tempo de pastejo.

Após obtenção dos dados, foram calculadas as médias das três repetições (BAGGIO et al., 2009), e de duas metodologias em teste, duas repetições (2R) e uma repetição (1R).

Foram avaliados três intervalos de observação, sendo a padrão (10 minutos) conforme metodologia de Silva et al. (2008), e duas metodologias em teste (20 e 30 minutos).
Foram avaliadas três metodologias do tempo total de avaliação, sendo uma com oito horas (08:00 às 15:50) e outra com 12 horas (06:00 às 17:50), comparadas com o tempo integral do dia (24 horas, 06:00 às 05:50). Entretanto foi considerado que as oito e 12 horas de avaliação foi durante o período diurno, pois essas metodologias são utilizadas com a justificativa de dificuldade de avaliação dos animais durante a noite.

Salienta-se que não há interesse em comparar todos os tratamentos em estudos, mas somente de comparar cada metodologia em teste com metodologias já validadas.

Os resultados foram interpretados estatisticamente por meio de análise de variância e teste Dunnett a 0,05 de probabilidade, através do programa estatístico SAEG (Versão 9.1). O modelo estatístico utilizado foi

$$
\text { Yijk }=\mu+\mathrm{Ti}+\text { eijk }
$$

em que: Yijk - o valor observado da variável; $\mu$ - constante geral;Ti - efeito do tratamento i; Eijk erro associado a cada observação.

\section{Resultados e Discussão}

No Tabela 2, verifica-se que os percentuais da atividade de pastejo da duração de avaliação $8 \mathrm{~h}$ e $12 \mathrm{~h}$ diferiram da duração de avaliação padrão de $24 \mathrm{~h}(\mathrm{P}<0,05)$. Foi verificado no presente estudo que os animais realizaram a atividade de pastejo com maior frequência durante o dia, sendo mais intensivos no início da manhã e no final da tarde. Com isso, o percentual de pastejo apresentou desproporcionalidade ao longo das 24 horas. Van Soest (1994) afirmou que o pastejo é mais intenso durante todo o período da manhã, portanto no presente estudo verificou-se elevada intensidade também no final da tarde. 
Tabela 2. Valores, em percentuais, para as atividades de pastejo, outras atividade, ruminação, alimentação no cocho, com duração de avaliação de 08 e 12 horas comparadas com o de 24 horas, de novilhas mestiças a pasto.

\begin{tabular}{lcccc}
\hline \multirow{2}{*}{ Item } & \multicolumn{3}{c}{ Duração de avaliação } & \multirow{2}{*}{ CV (\%) } \\
\cline { 2 - 4 } & 24 horas & 12 horas & 08 horas & \\
\hline PAS & 29,7 & $48,5^{*}$ & $42,1^{*}$ & 13,5 \\
OUT & 40,5 & 35,6 & 37,4 & 20,3 \\
RUM & 27,3 & $11,4^{*}$ & $13,9^{*}$ & 18,4 \\
COC & 2,5 & $4,5^{*}$ & $6,6^{*}$ & 8,4 \\
\hline
\end{tabular}

Médias seguidas por asterisco, na linha, diferem estatisticamente da duração de 24 horas, pelo teste Dunnett a 0,05 de probabilidade.

PAS - tempo de pastejo; OUT - tempo de outras atividades; RUM - tempo de ruminação; $\mathrm{COC}$ - tempo de alimentação no cocho.

${ }^{1}$ Coeficientes de variação.

Fonte: Elaboração dos autores.

Tendo em vista, a intensificação de uma atividade em determinados períodos ao longo do dia, a duração de avaliação de $8 \mathrm{~h}$ e $12 \mathrm{~h}$ apresentou maiores percentuais de pastejo $48,5 \%$ e $42,1 \%$, respectivamente.

A duração de avaliação em testes (8h e 12h) apresentou diferenças da duração de avaliação de 24 horas para os percentuais de ruminação $(\mathrm{P}<0,05)$. Uma maior frequência da atividade de ruminação no período noturno já tinha sido abordada por Damasceno, Baccari Junior e Targa (1999) e foi verificado também nesta avaliação. Sendo assim, as maiores frequências de ruminação ocorreram entre às 22:00 e 5:00 horas, o que impossibilita a avaliação de duração $8 \mathrm{~h}$ e $12 \mathrm{~h}$ em coletar a maior frequência da atividade, ocasionando diferença nos valores sendo $13,9 \%$ e $11,4 \%$ vs. $27,3 \%$ para $8 \mathrm{~h}$, $12 \mathrm{~h}$ e $24 \mathrm{~h}$, respectivamente.

Já para os percentuais de outras atividades, não foi verificado diferenças entre a duração de avaliação em testes e a padrão $(\mathrm{P}>0,05)$. Foi verificada uma distribuição irregular da atividade de outras atividades durante as 24 horas, sendo que em qualquer período de avaliação da atividade tende a apresentar valores em percentuais semelhantes.

Os percentuais da atividade alimentação no cocho para a duração de avaliação $8 \mathrm{~h}$ e $12 \mathrm{~h}$ apresentaram diferenças com a duração de avaliação de $24 \mathrm{~h}$ $(\mathrm{P}<0,05)$. Destaca-se que os animais demoraram, em média, um período de 30 minutos para o consumo do suplemento oferecido no dia, e o fornecimento foi realizado às 10 horas da manhã. Com isso, todas as metodologias detectaram o comportamento do consumo de concentrado (alimentação no cocho).

A atividade alimentação no cocho é uma atividade diferenciada das demais, pois o início da mesma depende do horário de fornecimento do suplemento, e só é exercido em um único período durante o dia, por isso, apresentou diferenças estatísticas devido as suas proporções, que foi em média de 30 minutos, em relação aos tempos de duração da avaliação, que foram $8 \mathrm{~h}, 12 \mathrm{~h}$ e $24 \mathrm{~h}$.

O somatório dos percentuais de pastejo e ruminação são próximos entre as diferentes durações de avaliação, apresentando valores de $57 \%, 59,9 \%$ e $56 \%$ para $24 \mathrm{~h}, 12 \mathrm{~h}$ e $8 \mathrm{~h}$, respectivamente. Com isso, pode-se afirmar que existe uma compensação entre as atividades de pastejo e ruminação, sendo a elevação de uma promove diminuição da outra.

Para o tempo de pastejo (PAS), ruminação (RUM), alimentação no cocho (COC), outras atividades (OUT), alimentação total (TAT) e mastigação total (TMT) não foram verificados diferenças estatísticas entre os intervalos de observações 20 e 30 minutos quando comparados com o de 10 minutos $(\mathrm{P}>0,05)$ (Tabela 3$)$. Esses resultados demonstram que para a coleta de dados dessas variáveis é possível aumentar o intervalo de observação, sem interferir na precisão dos dados. 
Tabela 3. Comportamento ingestivo com intervalos de observação de 20 e 30 minutos comparados com o de 10 minutos, de novilhas mestiças a pasto.

\begin{tabular}{lcccc}
\hline \multirow{2}{*}{ Item } & \multicolumn{3}{c}{ Intervalo de observação } & \multirow{2}{*}{ CV (\%) } \\
\cline { 2 - 4 } & 10 & 20 & 30 & \\
\hline PAS & 431 & 451 & 445 & 20,8 \\
OUT & 614 & 607 & 619 & 25,0 \\
RUM & 356 & 344 & 340 & 32,4 \\
COC & 39 & 38 & 36 & 39,0 \\
TAT & 469 & 489 & 481 & 19,0 \\
TMT & 826 & 833 & 821 & 18,6 \\
\hline
\end{tabular}

Médias seguidas por asterisco, na linha, diferem estatisticamente do intervalo de observação de 10 minutos, pelo teste Dunnett a 0,05 de probabilidade.

PAS - tempo de pastejo; OUT - tempo de outras atividades; RUM - tempo de ruminação; COC - tempo de alimentação no cocho; TAT - tempo de alimentação total; TMT - tempo de mastigação total.

${ }^{1}$ Coeficientes de variação.

Fonte: Elaboração dos autores.

Esses valores corroboram com os relatados de Silva et al. (2006, 2008), que constataram que qualquer intervalo entre cinco e 30 minutos entre as observações seria eficiente para estudar estas variáveis.

Não foram encontrados na literatura, trabalhos semelhantes a este estudo, que verificassem o efeito da duração de avaliação sobre os períodos do comportamento alimentar, não sendo possível realizar um paralelo ao presente estudo. Salientase a importância de mais estudos nesta área para comparação de metodologias que sejam adequados para descrever os fenômenos comportamentais com precisão e que possibilitem a redução da mão-deobra.

Os resultados similares do tempo de pastejo nos diferentes intervalos de observações sugerem que nas pesquisas que o objetivo é o tempo de pastejo, salientando-se a relação direta com as respostas animal como consumo, desempenho, eficiências e conversões alimentares, o intervalo de observação de 30 minutos é adequado, desta forma menor número de observadores poderão ser utilizados, lembrando que diminuirão em três vezes as observações (10 minutos para 30 minutos de intervalo de observação).

No estudo do comportamento ingestivo, o maior custo para execução é a mão-de-obra necessária para observação de um número adequado de animais. Sendo assim, tais resultados são de grande valia para a viabilização de estudos relacionados ao comportamento animal, pois, possibilitaria a observação de um maior número de animais, o que permite uma maior acurácia dos resultados, com um menor número de observadores, o que facilita a condução de estudos nesta área.

Por se tratar de estudos metodológicos, mais pesquisas devem ser realizadas em condições diversas para uma consolidação dessa metodologia em qualquer situação de avaliação do comportamento ingestivo de bovinos em pastejo.

Verifica-se na Tabela 4, que o número de períodos de pastejo (NPP), outras atividades (NPO) e ruminação (NPR), tempo por período de outras atividades (TPO) e ruminação (TPR), os intervalos de 20 e 30 minutos diferiram da escala de 10 minutos $(\mathrm{P}<0,05)$. Essa diferença encontrada muito provavelmente origina-se da consequente diminuição do número de observações nos intervalos de vinte e trinta minutos. Enquanto no intervalo de dez minutos foram colhidas 144 observações instantâneas, nos intervalos de vinte e trinta minutos foram 72 e 48 observações, respectivamente, o que aponta uma redução considerável de observações. 
Tabela 4. Períodos discretos do comportamento ingestivo com intervalos de observação de 20 e 30 minutos comparados com o de 10 minutos, de novilhas mestiças a pasto.

\begin{tabular}{lcccc}
\hline \multirow{2}{*}{ ITEM } & \multicolumn{3}{c}{ Intervalo de observação } & \multirow{2}{*}{ CV $^{1}(\%)$} \\
\cline { 2 - 4 } & 10 & 20 & 30 & \\
\hline NPP & 8,0 & $7,0^{*}$ & $5,9^{*}$ & 31,4 \\
NPO & 15,6 & $11,9^{*}$ & $9,9^{*}$ & 30,6 \\
NPR & 10,2 & $8,5^{*}$ & $7,0^{*}$ & 37,3 \\
NPC & 1,2 & 1,1 & 1,0 & 37,0 \\
TPP & 61,3 & 70,5 & $78,7^{*}$ & 36,0 \\
(mim) & & & & \\
TPO & 45,8 & $54,4^{*}$ & $66,4^{*}$ & 43,8 \\
(mim) & & & & \\
TPR & 38,5 & $42,6^{*}$ & $49,8^{*}$ & 29,9 \\
(mim) & & & 34,5 & 36,5 \\
TPC & 35,4 & 34,6 & 34,5 \\
(mim) & & & & \\
\hline
\end{tabular}

Médias seguidas por asterisco, na linha, diferem estatisticamente do intervalo de observação de 10 minutos, pelo teste Dunnett a 0,05 de probabilidade.

NPP - número de períodos de pastejo; NPO - número de períodos de outras atividades; NPR -número de períodos de ruminação; NPC - número de períodos de alimentação no cocho; TPP - tempo por período de pastejo; TPO - tempo por período de outras atividades; TPR - tempo por período de ruminação; TPC - tempo por período de alimentação no cocho. ${ }^{1}$ Coeficiente de variação.

Fonte: Elaboração dos autores.

O NPC e TPC não diferiram estatisticamente nos diferentes intervalos de observação avaliados $(\mathrm{P}>0,05)$. Embora muitos pesquisadores tenham optado por intervalos superiores à dez minutos para avaliar o comportamento ingestivo de animais ruminantes, observou-se neste estudo que essa opção torna-se inadequada quando se avalia o leque de variáveis, incluindo os períodos discretos de cada uma das atividades.

Para fazer uma avaliação mais detalhada do comportamento ingestivo, considerando o número de períodos discretos das atividades e o tempo de cada atividade, a escala de observação de dez minutos é mais exata e, portanto, é a recomendada, pois permite detectar melhor a freqüência diária de cada atividade, diminuindo as perdas de observações. Silva et al. (2006) relataram que intervalos superiores a dez minutos comprometeriam a precisão dos resultados de experimentos que visassem determinar as mesmas variáveis do presente estudo.

Verifica-se na Tabela 5, que todas as variáveis relacionadas às eficiências de alimentação e ruminação, não foram observadas diferenças entre os intervalos de 20 e 30 minutos com o intervalo de 10 minutos. Como as eficiências são valores calculados através do consumo dos nutrientes e o tempo de alimentação total ou ruminação, isso ocorreu em decorrência da não diferença estatística nos tempos de pastejo, ruminação e alimentação no cocho nos diferentes intervalos.

Tabela 5. Eficiência de alimentação e ruminação do comportamento ingestivo com intervalos de observação de 20 e 30 minutos comparados com o de 10 minutos, de novilhas mestiças a pasto.

\begin{tabular}{|c|c|c|c|c|}
\hline \multirow{2}{*}{ Item } & \multicolumn{3}{|c|}{ Intervalo de observação } & \multirow{2}{*}{$\mathrm{CV}^{1}(\%)$} \\
\hline & 10 & 20 & 30 & \\
\hline & \multicolumn{4}{|c|}{ Eficiência de alimentação (g/min) } \\
\hline EAMS & 12,2 & 11,7 & 12,0 & 22,3 \\
\hline EAFDN & 5,9 & 5,7 & 5,9 & 21,3 \\
\hline EANDT & 7,8 & 7,5 & 7,7 & 23,3 \\
\hline EACNF & 3,9 & 3,7 & 3,8 & 20,4 \\
\hline \multirow[t]{2}{*}{ EAPB } & 1,9 & 1,8 & 1,9 & 22,5 \\
\hline & \multicolumn{4}{|c|}{ Eficiência de ruminação (g/min) } \\
\hline ERMS & 17,2 & 17,9 & 18,4 & 37,2 \\
\hline ERFDN & 8,4 & 8,7 & 9,0 & 35,0 \\
\hline
\end{tabular}

Médias seguidas por asterisco, na linha, diferem estatisticamente do intervalo de observação de 10 minutos, pelo teste Dunnett a 0,05 de probabilidade.

EAMS - eficiência de alimentação de matéria seca; EAFDN - eficiência de alimentação de fibra em detergente neutro; EANDT - eficiência de alimentação de nutrientes digestíveis totais; EACNF - carboidratos não-fibrosos; EAPB - eficiência de alimentação de proteína bruta; ERMS - eficiência de ruminação de matéria seca; ERFDN - eficiência de ruminação de fibra em detergente neutro.

${ }^{1}$ Coeficientes de variação.

Fonte: Elaboração dos autores.

$\mathrm{Na}$ Tabela 6, as variáveis, número de mastigações merícicas por bolo (MMB), tempo por bolo ruminado (TBo), número de bolos ruminados 
por dia (BOL) e número de mastigações merícicas por dia (MMnd) não foram encontradas diferenças estatísticas entre os turnos de observação quando comparados com o MANTAR ( $\mathrm{P}>0,05)$.

Tabela 6. Aspectos da ruminação do comportamento ingestivo com observações no turno da manhã e da tarde, comparado com a média dos turnos (MANTAR), de novilhas mestiças a pasto.

\begin{tabular}{lcccc}
\hline \multirow{2}{*}{ Item } & \multicolumn{3}{c}{ Turno de observação } & \multirow{2}{*}{ CV $^{1}$ (\%) } \\
\cline { 2 - 5 } & MANTAR & MANHÃ & TARDE & \\
\hline MMB (n $\left.{ }^{\circ}\right)$ & 51,8 & 50,9 & 52,6 & 13,5 \\
TBo (seg.) & 48,7 & 49,8 & 47,6 & 13,5 \\
BOL (n $\left.{ }^{\circ}\right)$ & 443 & 440 & 447 & 37,7 \\
VeM (n $\%$ seg.) & 1,06 & $1,02^{*}$ & $1,11^{*}$ & 7,5 \\
TeM (seg.) & 0,94 & $0,98^{*}$ & $0,91^{*}$ & 7,7 \\
MMnd (n $\left.{ }^{\circ}\right)$ & 22545 & 22150 & 22940 & 34,9 \\
\hline
\end{tabular}

Médias seguidas por asterisco, na linha, diferem estatisticamente da observação em dois turnos (MANTAR), pelo teste Dunnett a $5 \%$ de probabilidade.

MMB - número de mastigações merícicas por bolo; TBo - tempo por bolo ruminado; BOL - número de bolos ruminados por dia; VeM - velocidade de mastigação; TeM - tempo por mastigação merícicas; MMnd - número de mastigações merícicas por dia. ${ }^{1}$ Coeficientes de variação.

Fonte: Elaboração dos autores.

$\mathrm{Na}$ literatura sobre comportamento ingestivo em pastejo (BREMM et al., 2008; ÍTAVO et al., 2008; FREITAS et al., 2010; GLIENKE et al., 2010; JOCHIMS et al., 2010; MENDES et al., 2010; MISSIO et al., 2010), verificou-se que essas quatro variáveis (MMB, TBo, BOL e MMnd) são as únicas utilizadas nas avaliações dos aspectos do comportamento ingestivo de ruminantes, esse resultado demonstra que não há necessidade de avaliação em dois turnos (manhã e tarde), sendo que somente uma avaliação, tanto pela manhã quanto pela tarde, apresenta resultados semelhantes.

A VeM e TeM apresentaram médias diferentes entre os turnos de observação $(\mathrm{P}<0,05)$. Para essas duas variáveis, a avaliação pela manhã e tarde (MANTAR) apresentou sempre valores intermediários entre a avaliação do turno da manhã e da tarde separadamente.

Na Tabela 7, o número de mastigação por bolo (MMB), tempo por bolo ruminado (TBo), número de bolos por dia (BOL), velocidade de mastigação (VeM), tempo por mastigação merícica (TeM) e número de mastigação merícica por dia (MMnd), a avaliação com apenas uma repetição (observação) por turno não diferiu estatisticamente da metodologia com três repetições por turno $(\mathrm{P}>0,05)$. Esse resultado demonstra que não há necessidade de três avaliações por turno, sendo que somente uma avaliação, apresenta resultados confiáveis.

Tabela 7. Aspectos da ruminação, deglutição e bocados do comportamento ingestivo com uma e duas repetições comparado com três repetições por observação, de novilhas mestiças a pasto.

\begin{tabular}{|c|c|c|c|c|}
\hline \multirow{2}{*}{ Variável } & \multicolumn{3}{|c|}{ Repetições por turno } & \multirow{2}{*}{$\mathrm{CV}^{1}(\%)$} \\
\hline & $3 \mathrm{R}$ & $2 \mathrm{R}$ & $1 \mathrm{R}$ & \\
\hline \multicolumn{5}{|c|}{ Aspectos da ruminação } \\
\hline $\operatorname{MMB}\left(n^{\circ}\right)$ & 51,8 & 51,8 & 51,4 & 13,8 \\
\hline TBo (seg.) & 48,7 & 49,0 & 49,1 & 12,8 \\
\hline $\operatorname{BOL}\left(n^{\circ}\right)$ & 447 & 433 & 427 & 37,0 \\
\hline VeM (n $\%$ seg.) & 1,06 & 1,06 & 1,05 & 7,1 \\
\hline $\mathrm{TeM}$ (seg.) & 0,94 & 0,95 & 0,96 & 7,1 \\
\hline MMnd $\left(n^{\circ}\right)$ & 22910 & 22025 & 21446 & 34,6 \\
\hline \multicolumn{5}{|c|}{ Aspectos da deglutição } \\
\hline $\operatorname{BDe}\left(\mathrm{n}^{\circ}\right)$ & 46,3 & 42,5 & $41,3^{*}$ & 10,1 \\
\hline TDe (min.) & 46,3 & 46,0 & 47,1 & 12,8 \\
\hline \multicolumn{5}{|c|}{ Aspectos do bocado } \\
\hline TxB (n $/$ seg.) & 0,88 & 0,93 & $1,01^{*}$ & 8,83 \\
\hline MaB (g MS) & 0,20 & 0,19 & 0,17 & 27,9 \\
\hline $\operatorname{NBD}\left(\mathrm{n}^{\circ}\right)$ & 15615 & 16622 & 17846 & 27,2 \\
\hline
\end{tabular}

Médias seguidas por asterisco, na linha, diferem estatisticamente de três repetições por turno, pelo teste Dunnett a 0,05 de probabilidade.

MMB - número de mastigações merícicas por bolo; TBo - tempo por bolo ruminado; BOL - número de bolos ruminados por dia; VeM - velocidade de mastigação; TeM - tempo por mastigação merícica; MMnd - número de mastigações merícicas por dia; BDe - números de bocados por deglutição; TDe - tempo por deglutição; TxB - taxa de bocado; MaB - massa de bocado; NBD - número de bocados por dia.

${ }^{1}$ Coeficientes de variação.

Fonte: Elaboração dos autores. 
Diante da escassez de estudos com esta finalidade, torna-se mais lento a consolidação da metodologia padrão. Com isso, deverão ser realizados mais estudos de metodologia dos aspectos do comportamento ingestivo com o intuito de verificar quais as quantidades de avaliações que são realmente essenciais para obter os resultados confiáveis. Outra razão seria a diminuição da mãode-obra gasta na condução desses estudos, uma vez que apresentam altos custos.

$\mathrm{O}$ BDe e a TxB apresentaram valores estatisticamente diferentes entre a $1 \mathrm{R}$ e $3 \mathrm{R}(\mathrm{P}<0,05)$. No entanto, para os mesmos números de repetições, as médias encontradas para TDe, MaB e NBD foram estaticamente semelhantes $(\mathrm{P}>0,05)$. Com isso, verifica-se que o uso de uma repetição (1R), não pode ser validada pelo motivo de não apresentar confiabilidade devido à diferença que provoca nas médias das variáveis em estudo.

Já o uso de duas repetições (2R) apresentou valores estatisticamente iguais quando comparados com os resultados de três repetições (3R) $(\mathrm{P}>0,05)$. Por tanto, verificou-se que para estudos de avaliação do bocado e de deglutição em bovinos, a metodologia com duas repetições apresentou valores confiáveis, o que possivelmente provocará uma diminuição da necessidade de mão-de-obra e materiais, obtendo como consequência uma minimização dos custos para execução destes estudos.

\section{Conclusão}

A duração de avaliação de 24 horas é a recomendada para avaliação do comportamento ingestivo em termos de parâmetros nutricionais e metabólicos, pois as demais promovem distorção dos dados em função da variação da intensidade das atividades ao longo do dia. Com exceção dos períodos discretos das atividades comportamentais, o estudo do comportamento pode ser realizado em intervalos com até 30 minutos, sem interferir na precisão dos resultados. Para avaliação dos aspectos da ruminação do comportamento ingestivo, recomenda-se a utilização de um turno de avaliação podendo ser pela manhã ou tarde, sendo que a mesmo poderá ser com apenas uma repetição. Recomendase a utilização de pelo menos duas repetições no estudo dos bocados de novilhas suplementadas a pasto.

\section{Referências}

BAGGIO, C.; CARVALHO, P. C. F.; SILVA, J. L. S.; ANGHINONI, I.; LOPES, M. L. T.; THUROWET, J. M. Padrões de deslocamento e captura de forragem por novilhos em pastagem de azevém-anual e aveia-preta manejada sob diferentes alturas em sistema de integração lavoura-pecuária. Revista Brasileira de Zootecnia, Viçosa, MG, v. 38, n. 2, p. 215-222, 2009.

BREMM, C.; ROCHA, M. G.; FREITAS, F. K.; MACARI, S.; ELEJALDE, D. A. G.; ROSO, D. Comportamento ingestivo de novilhas de corte submetidas a estratégias de suplementação em pastagens de aveia e azevém. Revista Brasileira de Zootecnia, Viçosa, MG, v. 37, n. 7, p. 11611167, 2008.

BURGER, P. J. PEREIRA, J. C.; QUEIROZ, A. C.; SILVA, J. F. C.; VALADARES FILHO, S. C.; CECON, P. R.; CASALI, A. D. P. Comportamento ingestivo de bezerros holandeses alimentados com dietas contendo diferentes níveis de concentrado. Revista Brasileira de Zootecnia, Viçosa, MG, v. 29, n. 1, p. 236-242, 2000.

DAMASCENO, J. C.; BACCARI JUNIOR, F.; TARGA, L. A. Respostas comportamentais de vacas holandesas, com acesso à sombra constante ou limitada. Pesquisa Agropecuária Brasileira, Brasília, v. 34, n. 4, p. 709-715, 1999.

FREITAS, L. S.; SILVA, J. H. S.; SEGABINAZZI, L. R.; SILVA, V. S.; ALVES FILHO, D. C.; BRONDANI, I. L. Substituição da silagem de milho por silagem de girassol na dieta de novilhos em confinamento: comportamento ingestivo. Revista Brasileira de Zootecnia, Viçosa, MG, v. 39, n. 1, p. 225-232, 2010.

GARY, L. A.; SHERRITT, G. W.; HALE, E. B. Behavior of charolais cattle on pasture .Journal of Dairy Science, Madison, v. 30, n. 2, p. 303- 306, 1970.

GLIENKE, C. L.; ROCHA, M. G.; ROSO, D.; POTTER, L.; COSTA, V. G.; MACHADO, J. M. Ingestive behavior and displacement patterns of beef heifers on italian ryegrass pasture. Revista Brasileira de Zootecnia, Viçosa, MG, v. 39, n. 2, p. 247-254, 2010.

HANCOCK, J. Grazing behaviour of cattle. Animal Breeding, Abstract, Wallingford, v. 21, n. 1, p. 1-13, 1953. 
HODGSON, J. Ingestive behavior. In: LEAVER, J. D. (Ed.). Herbage intake handbook. Hurley: British Grassland Society, 1982. p. 113-138.

ÍTAVO, L. C. V.; SOUZA, S. R. M. B. O.; RÍMOLI, J.; ÍTAVO, C. C. B. F.; DIAS, A. M. Comportamento ingestivo diurno de bovinos em pastejo contínuo e rotacionado. Archivos de Zootecnia, Córdoba, v. 57, n. 217, p. 43-52, 2008.

JAMIESON, W. S.; HODGSON, J. The effect of variation in sward characteristics upon the ingestive behavior and herbage intake of calves and lambs under continuous stocking management. Grass and Forage Science, Oxford, v. 34, n. 4, p. 273-281, 1979.

JOCHIMS, F.; PIRES, C. C.; GRIEBLER, L.; BOLZAN, A. M. S.; DIAS, F. D.; GALVANI, D. B. Comportamento ingestivo e consumo de forragem por cordeiras em pastagem de milheto recebendo ou não suplemento. Revista Brasileira de Zootecnia, Viçosa, MG, v. 39, n. 3, p. 572-581, 2010.

MACARI, S.; ROCHA, M. G.; POTTER, L.; ROMAN, J.; BREMM, C.; COSTA, V. G. Comportamento ingestivo diurno de novilhas de corte recebendo níveis de suplemento. Ciência Rural, Santa Maria, v. 37, n. 6, p. 1746-1752, 2007.

MENDES, C. Q.; TURINO, V. F.; SUSIN, I., PIRES, A. V.; MORAES, J. B.; GENTIL, R. S. Comportamento ingestivo de cordeiros e digestibilidade dos nutrientes de dietas contendo alta proporção de concentrado e diferentes fontes de fibra em detergente neutro. Revista Brasileira de Zootecnia, Viçosa, MG, v. 39, n. 3, p. 594600, 2010.

MISSIO, R. L.; BRONDANI, I. L.; ALVES FILHO, D. C.; SILVEIRA, M. F.; FREITAS, L. S.; RESTLE, J. Comportamento ingestivo de tourinhos terminados em confinamento, alimentados com diferentes níveis de concentrado na dieta. Revista Brasileira de Zootecnia, Viçosa, MG, v. 39, n. 7, p. 1571-1578, 2010.

OLIVO, C. J.; CHARÃO, P. S.; ZIECH, M. F.; ROSSAROLLA, G.; MORAES, R. S. Comportamento de vacas em lactação em pastagem manejada sob princípios agroecológicos. Revista Brasileira de Zootecnia, Viçosa, MG, v. 35, n. 6, p. 2443-2450, 2006.
PARDO, R. M. P.; FISCHER, V.; BALBINOTTI, M.; ZANELA, M. B.; MORENO, C. B.; FERREIRA, E. X.; VINHAS, R. I.; MONKS, P. L. Comportamento ingestivo diurno de novilhos em pastejo a níveis crescentes de suplementação energética. Revista Brasileira de Zootecnia, Viçosa, MG, v. 32, n. 6, p. 1408-1418, 2003.

PEREIRA, L. M. R.; FISCHER, V.; MORENO, C. B.; PARDO, R. M. P.; GOMES, J. F.; MONKS, P. L. Comportamento ingestivo diurno de novilhas Jersey em pastejo recebendo diferentes suplementos. Revista Brasileira de Agrociência, Jaboticabal, v. 11, n. 4, p. 453459, 2005.

SILVA, R. R.; CARVALHO, G. G. P.; MAGALHÃES, A. F.; SILVA, F. F.; PRADO, I. N.; FRANCO, I. L.; VELOSO, C. M.; CHAVES, M. A.; PANIZZA, J. C. J. Comportamento ingestivo de novilhas mestiças de Holandês em pastejo. Archivos de Zootecnia, Córdoba, v. 54, n. 205, p. 63-74, 2005.

SILVA, R. R.; SILVA, F. F.; PRADO, I. N.; CARVALHO, G. G. P.; FRANCO, I. L.; ALMEIDA, V. S.; CARDOSO, C. P.; RIBEIRO, M. H. S. Comportamento ingestivo de bovinos. Aspectos metodológicos. Archivos de Zootecnia, Córdoba, v. 55, n. 211, p. 293-296, 2006.

SILVA, R. R.; PRADO, I. N.; CARVALHO, G. G. P.; SANTANA JUNIOR, H. A.; SILVA, F. F.; DIAS, D. L. S. Efeito da utilização de três intervalos de observações sobre a precisão dos resultados obtidos no estudo do comportamento ingestivo de vacas leiteiras em pastejo. Ciência Animal Brasileira, Goiânia, v. 9, n. 2, p. 319326, 2008.

SILVA, R. R.; PRADO, I. N.; SILVA, F. F.; ALMEIDA, V. V. S.; SANTANA JUNIOR, H. A.; QUEIROZ, A. C.; CARVALHO, G. G. P.; BARROSO, D. S. Comportamento ingestivo diurno de novilhos nelore recebendo níveis crescentes de suplementação em pastejo de capimbraquiária. Revista Brasileira de Zootecnia, Viçosa, MG, v. 39, n. 9, p. 2073-2080, 2010.

SOUZA, S. R. M. B. O.; ÍTAVO, L. C. V.; RÍMOLI, J.; ÍTAVO, C. C. B. F.; DIAS, A. M. Comportamento ingestivo diurno de bovinos em confinamento e em pastagens. Archivos de Zootecnia, Córdoba, v. 56, n. 213, p. 67-70, 2007.

VAN SOEST, P. J. Nutritional ecology of the ruminant. 2. ed. New York: Cornell University Press, 1994. 476 p. 
